\title{
Les tableaux de bord de gestion : leur utilité et leurs limites
}

\author{
François Bernard Malo, Université Laval \\ Rita Labadie et René Rouleau, Centre hospitalier universitaire de Québec ${ }^{1}$
}

Votre enfant en pleurs vient vous rejoindre en plein milieu de la nuit. Comme vous vous apprêtez à lui raconter une histoire drôle, pensant qu'il n'a fait qu'un mauvais rêve, vous constatez avec effroi que son front est terriblement chaud! Après avoir sorti un thermomètre de la pharmacie et l'avoir mis sous sa langue, vos pires craintes se confirment : votre enfant a vraisemblablement une infection et sa température anormalement élevée n'est qu'une indication qu'il va falloir rencontrer un médecin au plus vite.

En tant que gestionnaire, vous vous dites sûrement que, s'il était possible de mesurer avec précision la « température organisationnelle » de votre entreprise, ce serait fantastique. On pourrait ainsi savoir quand consulter un spécialiste et, de ce fait, sauver annuellement des milliers de dollars en frais inutiles.

Eh bien, vous savez quoi ? Les « thermomètres organisationnels » existent bel et bien! Seulement, ils ne se vendent pas tout faits... Il vous faudra le construire. Comment? C'est exactement ce que cet article tâchera de vous démontrer.

Le « thermomètre organisationnel » est appelé par les spécialistes en gestion «tableau de bord de gestion (TBG) ». Outil d'aide à la prise des décisions inventé en France dans les années 1930, ce n'est que depuis les années 1980 qu'il a commencé à se populariser de ce côté-ci de 1'Atlantique. Se référant à Ponthière ${ }^{2}$, Malo $^{3}$ explique que tout bon TBG doit être synoptique et rassembler en un nombre limité de coups d'œil toutes les informations nécessaires au pilotage quotidien de l'entreprise. Sous la forme de suivi de ratios et de graphiques nécessaires au décideur pour prendre de bonnes décisions, le TBG peut comporter une vingtaine d'indicateurs de performance dans le cas d'une petite organisation et près d'une cinquantaine dans le cas d'une organisation de plus grande taille.

De l'avis des spécialistes, un tel outil ne saurait être « vendu » à des milliers d'exemplaires indifférenciés et cela, pour une raison bien simple. Afin d'être véritablement utile, le TBG doit être adapté à la réalité de chaque organisation et traduire en indicateurs précis la stratégie choisie par les dirigeants. «Décliner la stratégie concrètement, tel est l'un des objectifs majeurs assignés [...] au tableau de bord. » ${ }^{4}$ Pour réussir cette traduction, il convient de procéder en deux temps.

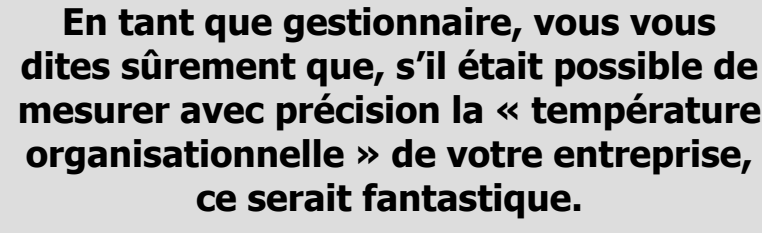
s'assurer d'une bonne position sur leur marché des produits et des services. Misant sur leurs forces tout en profitant des opportunités qui se présentent dans leur environnement externe ${ }^{5}$, les gestionnaires devront se fixer des objectifs (à court, moyen et long termes), puis ensuite déterminer les facteurs clés de succès sur lesquels il faudra agir pour atteindre ces derniers.

Dans un deuxième temps, après avoir précisé les centres de responsabilités impliqués par cette stratégie, les gestionnaires devront chercher à identifier quels indicateurs pourront éventuellement servir à mesurer l'état de la situation et ultimement l'atteinte des objectifs fixés au préalable. À cette deuxième étape, il est souvent utile de se donner des normes de référence inspirées de ce que font les meilleurs dans notre secteur d'activité ${ }^{6}$. 
Pour Kaplan et Norton ${ }^{7}$, quatre questions peuvent aider les gestionnaires de toutes sortes d'organisations à construire leur propre TBG : pour réussir financièrement, qu'attendent de nous nos actionnaires ? Pour réussir notre ambition, comment devons-nous apparaître à nos clients ? Pour satisfaire nos actionnaires et nos clients, quels processus clés devons-nous maîtriser? Et enfin, pour réaliser notre ambition, comment pouvons-nous développer notre capacité à nous améliorer?

Comme vous l'aurez sans doute constaté, ces quatre questions renvoient implicitement à prendre en considération quatre dimensions incontournables dans l'analyse des performances organisationnelles: la dimension financière (propre aux actionnaires), la dimension clientèle (propre à notre société capitaliste où le client est roi), la dimension des processus internes (propre aux gestionnaires de l'organisation) et la dimension "croissance et développement» (propre aux ressources humaines évoluant au sein de l'organisation).

Une fois votre propre TBG construit et rendu opérationnel, vous aurez donc entre les mains un outil extrêmement utile pour vous alerter de la présence d'écarts par rapport aux prévisions et pour identifier des opportunités qui mériteraient peut-être que vous révisiez en partie votre plan stratégique. Ceci dit, vous demandez-vous peut-être, est-ce que ça marche vraiment?

Il y a peu de temps Kaplan ${ }^{8}$ démontrait, chiffres à l'appui, comment l'unité de soins intensifs pédiatriques du Duke Children's Hospital avait implanté avec succès un TBG qui avait contribué à faire en sorte que, d'une situation déficitaire de l'ordre de 50 millions de dollars, on était passé à une situation où, trois ans plus tard, on enregistrait un bénéfice de près de 10 millions de dollars. Plus près de nous, le Centre hospitalier universitaire de Québec (CHUQ) s'est aussi engagé récemment dans la mise sur pied d'un TBG. Bien que l'expérience en soit encore à ses débuts, tout nous porte à croire que, depuis sa mise en place, les gestionnaires sont mieux équipés que par le passé pour faire leur travail et prendre les bonnes décisions ${ }^{9}$.

Question de ne pas laisser sous-entendre que les TBG pourraient être une recette-miracle facile à appliquer

et qui marcherait à tout coup, voici quelques conseils qui pourraient vous éviter bien des ennuis.

Tout d'abord, n'essayez pas de mettre au point un TBG sans, au préalable, avoir obtenu un engagement ferme de la haute direction à l'effet qu'elle vous appuie dans cette démarche et qu'elle vous soutiendra en cas de besoin. Deuxièmement, n'essayez pas de mettre au point un TBG sans nommer un responsable qui devra périodiquement rendre des comptes sur l'évolution du dossier. Troisièmement, une fois le leader du projet sélectionné, il est très important que celui-ci adopte une démarche participative structurée, logique et continue. En effet, a fortiori dans les grandes organisations, il est pratiquement impossible d'imposer l'usage d'un TBG à des gestionnaires qui n'en n'ont jamais entendu parler.

Quatrièmement, le leader du projet doit sans cesse motiver les troupes à continuer de participer à la conception et à l'utilisation du TBG car, force nous est d'admettre que les organisations où une authentique préoccupation pour la mesure des performances organisationnelles globales existe réellement sont plutôt rares.

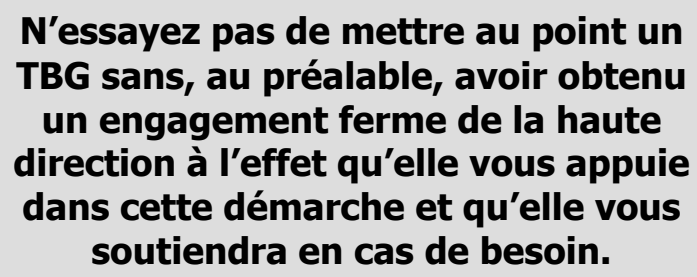

Cinquièmement, afin de pouvoir corriger le tir de façon continue et ainsi éviter les écueils importants, le leader du projet aura tout avantage à procéder de manière incrémentale (approche de «projet-pilote» ou démarche à petits pas) et à permettre à tous les utilisateurs éventuels de participer activement au processus de réflexion et de décision entourant le choix des indicateurs servant à mesurer les différents aspects des performances de l'organisation.

Enfin, sixièmement, un support technique informatique efficace et une mise à jour respectueuse des ressources à consacrer constituent un dernier élément à ne pas oublier. En effet, à quoi servirait un TBG dont les coûts d'utilisation et d'entretien dépasseraient les bénéfices? Toujours dans cette veine, nous 
croyons également que le projet de mise sur pied d'un TBG au sein d'une organisation doit parvenir à produire assez tôt des bénéfices pour l'organisation et les personnes qui y œuvrent. Sans cela, le découragement risque bien de venir saborder l'ensemble du projet.

Maintenant que vous êtes tous convaincus de l'utilité de mettre au point votre propre TBG afin de mesurer vous-mêmes votre "température organisationnelle ", voici une dernière chose à ne pas oublier: aucun TBG, aussi efficace soit-il, ne saurait se substituer à un bon gestionnaire. Au même titre qu'un marteau dans les mains d'un ouvrier malhabile ne donnerait rien qui vaille, un bon TBG sans gestionnaire professionnel pour l'analyser et y faire reposer ses décisions ne servirait à rien de constructif.

\section{Notes et références}

1 Détenteur d'un doctorat en sciences de gestion de l'Université de Toulouse 1, François Bernard Malo est professeur de gestion des ressources humaines au Département des relations industrielles de l'Université Laval. Rita Labadie est infirmière et conseillère à la direction générale au Centre hospitalier universitaire de Québec. René Rouleau est le directeur général du CHUQ.
2 Ponthière, M. (1935), dans Lauzel et Cibert (1959), Le bureau moteur, p. 143 (référence incomplète).

3 Malo, J.-L. (1995), «Tableau de bord», Encyclopédie de gestion, p. 923-938.

4 Mendoza, C., et Zhiren, R. (1999). «Le tableau de bord: en V.O. ou en version américaine? », Revue française de comptabilité, $\mathrm{n}^{\circ} 309$, p. 62.

5 Plusieurs spécialistes recommandent aussi de ne pas oublier de prendre en compte les faiblesses de l'organisation de même que les menaces présentes dans son environnement externe.

6 Cette idée fait référence à ce que l'on appelle le benchmarking.

7 Kaplan, R.S., et Norton, D.P. (1992). «The BalancedScorecard - Measures that Drive Performance », Harvard Business Review, janvier-février, p. 71-79.

8 Kaplan, R.S. et Norton, D.P. (2001). Comment utiliser le tableau de bord prospectif, Paris, Éd. d'Organisation.

9 Le lecteur intéressé par de plus amples démonstrations de l'apport des TBG à l'amélioration des performances organisationnelles globales aura avantage à lire la contribution de Lawson, Stratton et Hatch (2003). "The Benefits of a Scorecard System », CMA Management, juin/juillet, p. 2426. 


\section{Publicité}

\section{VRM}

